\title{
Re-imagining Healthcare: The Northwest Territories Transitions to an Integrated Chronic Disease Management Strategy
}

\author{
Erin Leith, Christine Kirvan, Jennifer Y. Verma, Kay Lewis and Scott Robertson
}

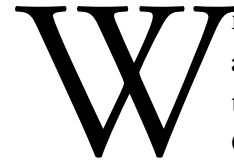

ith the goal to improve healthcare services and delivery, Stanton Territorial Hospital in the Northwest Territories (NWT) invited the Canadian Health Services Research Foundation (CHSRF) to explore opportunities to collaborate in the redesign and improvement of the health system. This article describes the NWT's efforts to transform its healthcare delivery to more appropriately care for people living with chronic diseases. It also sets the context for the initial meeting between the NWT and CHSRF and the collaborative journey that continues, with the bringing together of 40 health system managers, policy makers and clinicians, along with CHSRF staff and guest faculty, with a shared vision to redesign care to better meet the needs of people living with chronic diseases in the territory.

In the NW'T, chronic diseases account for approximately $70 \%$ of all deaths and half of all hospital admission days (Northwest Territories Department of Health and Social Services [DHSS] 2011b). As elsewhere, it is understood that healthcare services are not currently meeting population needs. Canadian health systems rank poorly in international comparisons that focus on the delivery of primary care, especially to those with multiple chronic conditions (Schoen et al. 2009). It is also well recognized in Canada that few healthcare systems are patient centred. In a recently commissioned CHSRF synthesis on transformation, professors Ross Baker and Jean-Louis Denis (2011) remarked, "Healthcare systems tend to reproduce their dominant logic and consequently neglect other areas where major care deficits persist." In another CHSRF-commissioned synthesis on transformation, Professor Denis and colleagues (2011) noted that "a clear vision and a coherent set of strategies are required to transform the system and achieve better alignment between the care offered and the care the population needs today." Such is the challenge for the NWT.

In its recently released 2011-2016 strategic plan, DHSS (2011a) set a priority to "improve the health status of the population" through the development of a culturally appropriate chronic disease management model that tracks system quality outcome measures. With a senior leadership dedicated to improving the health status of its population as a key strategic priority and highly engaged throughout the change manage- ment process, the NWT is well positioned to improve healthcare services and delivery through transformation. CHSRF, in partnership with NWT leadership, intends to support the territory through its longer-term vision, to strategically reorient the health system for the benefit of all those living in the territory. We also hope that the lessons we learn here hold value for learning elsewhere.

\section{Unique Challenges to Delivering Health Services in the NWT}

Informed by key territory health leaders, an informal needs assessment carried out by CHSRF in June 2010 revealed unique challenges faced by those who develop health policy and deliver health services in the NWT. Eight regional health authorities together with DHSS deliver health services to a diverse population of 45,000 northerners with a number of unique health needs across a sizeable geographical area that includes 33 communities, most with no road access. Staff turnover is extremely high in the NWT, resulting in an above-average number of short-term healthcare providers - often from southern provinces. All of this poses a significant challenge to building and sustaining the capacity to deliver consistent and quality care across the NWT.

At the time of the needs assessment, the auditor general of Canada had conducted an audit of DHSS's ability to manage the provision of health services, identify priorities and set directions for the health system, as well as its capacity to monitor, assess and report on results. The auditor general (2011) found that although DHSS has identified a number of areas for health system improvement, performance indicators to monitor progress were not yet in place. DHSS wholly agreed with the auditor general's findings and recommendations and has since used the report as a leverage point to spur health system improvement initiatives.

Chronic disease is prevalent across the NWT, with approximately 200 new cases of diabetes diagnosed each year. Where mental health is concerned, $64 \%$ of the population rate their mental health as excellent or very good, compared with $73 \%$ of other Canadians. Seniors aged 60 years and older are the fastest-growing segment of the NWT population, with this age group expected to increase from $9 \%$ in 2008 to $13 \%$ by 2017 . 
Given that the incidence of chronic disease increases with age, DHSS is expecting a significant impact on the demand, use and cost of health services. All of these add strain to an already-stretched healthcare system that consumes more than $25 \%$ of the territorial government budget $-\$ 326$ million in 2010-2011 (DHSS 2011b).

The needs assessment and auditor general's report were critical in informing and laying the foundation for a supportive partnership between CHSRF and DHSS for health system improvement and redesign.

\section{Collaboration for Innovation and Improvement}

Encouraged by the preliminary discussions with NWT health leadership, CHSRF staff and guest faculty set out to develop a tailored process to support the development of a territorial integrated chronic disease management strategy. By reflecting upon the lessons learned about organizational improvement from the Executive Training for Research Application (EXTRA) program and by consulting experts in change management, CHSRF identified a number of criteria that, if in place, would help to ensure the feasibility and success of the improvement effort. Ideally the strategy should

- be a priority for the organization/system,

- reflect a system-level issue,

- be innovative and identify effective and sustainable strategies,

- be actionable, practical and effective,

- include channels for the spread or systematic uptake across systems,

- provide opportunities to scale up improvements,

- build capacity for sustained health system improvement,

- impact on/be of importance to multiple stakeholders and

- use evidence, best practice and local expertise.

Fulfilling all of these criteria, CHSRF and DHSS formally agreed to partner toward the development of a territorial integrated chronic disease management strategy in the fall of 2010. Over the past year, CHSRF developed and delivered a series of four workshops designed to guide interdisciplinary cross-regional teams of health system managers, policy makers and healthcare providers in the co-design of health system improvement plans focused on key areas of chronic disease, while also prioritizing a systems perspective (e.g., an approach that allowed for small-scale improvements that could be scaled up as the collaboration progressed). DHSS staff felt it was most critical to prioritize mental health, diabetes and renal disease all high-burden disease areas - and that these specific chronic conditions could serve as tracers. This tracer approach allowed teams to pinpoint cross-cutting themes, issues and solutions that will ultimately be used to scale-up the improvements to meet the needs for the wider population.

CHSRF designed workshops that aimed to build capacity for participants to describe and understand health system problems, identify solutions and articulate reasoned improvement plans based on shared experience, regional evidence and data, and grounded within national and international best practices. The deputy minister also saw this as an opportunity to challenge participants to "re-imagine" - to think outside the constraints of the existing system and re-imagine a system that would best meet the needs of northerners.

\section{Early Results}

The evaluative approach that CHSRF brought to the NWT collaboration focuses, in our initial work together, on measuring key performance outcomes of the workshop participants (e.g., to what extent is CHSRF helping NWT health system leaders gain the skills in evidence use to address their healthcare challenges?). Early results demonstrate that participants valued the evidenceinformed approach provided, with $89 \%$ recommending this process to other organizations. Participants appreciated the opportunity to partake in an interdisciplinary, team-based setting that brought together a unique mixture of chronic disease management professionals and researchers, with CHSRF staff as brokers. This method helped to break down silos within the department and across the NWT, to foster multiple perspectives and to identify common problems and gaps across the health system. As a result, participants grew to think of chronic disease management as a system issue, rather than being disease specific. For example, one participant stated, "A key indicator of success for all of us is that now we think much more in terms of the system and how we're interconnected and how some of the work that goes on here needs to be integrated in the work that we do as individuals. We have really evolved to think of the system as a whole, rather than as individuals."

Workshop participants described their enhanced capacity in using evidence as the major enabling factor for the successful implementation of their pilot projects. This stemmed from the belief that participants had become champions for change who would continue to foster greater engagement and facilitate the uptake of the proposed initiatives. In the continued collaborative work, the evaluative plan will focus more specifically on the health, healthcare and economic impacts of the change that were informed through the workshops. Consideration for how the lessons learned (evidence and ideas) may be spread within the region and, where appropriate, across Canada is also a priority.

\section{NWT Improvement Plan Highlights}

CHSRF and DHSS will continue to collaborate through the implementation and evaluation of four improvement pilots that will inform the development of a territorial integrated chronic disease management strategy: 
1. Mental health

- Develop standardized processes for sharing client information between providers

- Establish standardized minimum competencies and service levels for mental health programs and providers

2. Diabetes

- Integrate a self-management approach as a component for programs and services

3. Renal disease

- Establish a process for a consistent single entry point to the renal care system

- Develop a territorial database to determine baseline data

- Develop an algorithm for primary care decision support of early-stage renal disease detection and management

4. System level

- Develop an integrated chronic disease prevention and management framework as the foundation to create chronic care program standards

The development of the pilots represents a key and measurable milestone toward the DHSS's priority to "improve the health status of the population." The pilots are also a critical step in the journey toward a territory-wide integrated chronic disease management strategy. The lessons learned will provide essential insight and evaluative evidence to support the further spread and scaling up of the NWT chronic disease management strategy.

CHSRF will continue to provide strategic advice and guidance throughout the implementation phase, providing ongoing change management coaching and developing and delivering two learning workshops designed to guide the improvement teams in the development of stakeholder engagement strategies and evaluation indicators. These essential components will add to the well-developed improvement plans and support timely reporting to address the DHSS strategic plan priorities and auditor general's recommendations.

\section{Re-imagining Healthcare Requires Committed Leadership and Collaboration}

Regardless of whether an innovation comes from the front line or emerges as a broad-scale improvement effort, high-performing systems share common characteristics. Recognizing organizations as the source site of innovation, Baker and Denis (2011) identify 10 themes underpinning the creation and sustainability of high-performing systems, beginning with engaged strategic leadership that prioritizes the development of lasting local capacity and the skill to support improvement, a team-based approach and the engagement of patients in the design and seamless integration of care.

CHSRF is an independent organization dedicated to accelerating healthcare improvement and transformation for Canadians. We collaborate with governments, policy makers and health system leaders to convert evidence and innovative practices into actionable policies, programs, tools and leadership development. Through our Collaboration for Innovation and Improvement program, we respond to regional, provincial and territorial health priorities and aim to develop and mobilize local capacity to assess, design, implement, evaluate and spread innovation. For more information about our work, please visit www.chsrf.ca. HQ

\section{References}

Auditor General of Canada. 2011. Report of the Auditor General of Canada to the Northwest Territories Legislative Assembly - March 2011. Ottawa, ON: Office of the Auditor General of Canada.

Baker, G.R. and J.L. Denis. 2011. A Comparative Study of Three Transformative Healthcare Systems: Lessons for Canada. Ottawa, ON: Canadian Health Services Research Foundation. Retrieved December 15, 2011. <http://www.chsrf.ca/Libraries/Commissioned_Research_ Reports/Baker-Denis-EN.sflb.ashx>.

Denis, J.L., H.T.O. Davies, E. Ferlie and L. Fitzgerald. 2011. Assessing Initiatives to Transform Healthcare Systems: Lessons for the Canadian Healthcare System. Ottawa, ON: Canadian Health Services Research Foundation. Retrieved December 15, 2011. <http://www.chsrf.ca/PublicationsAndResources/ResearchReports/ CommissionedResearch/11-07-21/c70d5dc2-98b6-43cb-b735e03144d0cf7a.aspx>.

Northwest Territories Health and Social Services. 2011a. Building on Our Foundation 2011-2016: A Strategic Plan for the NWT Health and Social Services System. Yellowknife, NT: Government of Northwest Territories. Retrieved December 15, 2011. <http://www.hlthss.gov. nt.ca/pdf/reports/health_care_system/2011/english/building_on_our_ foundation.pdfs.

Northwest Territories Health and Social Services. 2011b. Northwest Territories Health Status Report. Yellowknife, NT: Government of Northwest Territories. Retrieved December 15, 2011.<http://www. hlthss.gov.nt.ca/pdf/reports/health_care_system/2011/english/nwt_ health_status_report.pdf>.

Schoen, C., R. Osborn, M.M. Doty, D. Squires, J. Peugh and S. Applebaum. 2009. "A Survey of Primary Care Physicians in 11 Countries, 2009: Perspectives on Care, Costs, and Experiences." Health Affairs 28(6):w1171-83. Epub 2009 Nov 2. Retrieved December 15, 2011. <http://www.commonwealthfund.org/Publications/In-theLiterature/2009/Nov/A-Survey-of-Primary-Care-Physicians.aspx>.

\section{About the Authors \\ Erin Leith is the senior advisor of collaboration for innovation and improvement at the Canadian Health Services Research Foundation (CHSRF), in Ottawa, Ontario.}

Christine (Kirby) Kirvan is the program officer for collaboration for innovation and improvement at CHSRF.

Jennifer Y. Verma is the director of collaboration for innovation and improvement at CHSRF.

Kay Lewis is the chief executive officer of Stanton Territorial Health Authority, in Yellowknife, Northwest Territories.

Scott Robertson is the chief nursing officer for the Department of Health and Social Services in the Government of the Northwest Territories. 